\title{
A branched luminescent multinuclear platinum(II) complex
}

\author{
Hui Yang, ${ }^{1, a)}$ S. J. Xu, ${ }^{1, b)}$ Chi-Hang Tao, ${ }^{2}$ Vivian Wing-Wah Yam, ${ }^{2, c)}$ and Jie Zhang ${ }^{3}$ \\ ${ }^{1}$ Department of Physics, The University of Hong Kong, Pokfulam Road, Hong Kong, \\ People's Republic of China \\ ${ }^{2}$ Institute of Molecular Functional Materials (Areas of Excellence Scheme, University Grants Committee \\ (Hong Kong)), and Department of Chemistry, The University of Hong Kong, Pokfulam Road, Hong Kong, \\ People's Republic of China \\ ${ }^{3}$ Laboratory of Optical Physics, Institute of Physics, Chinese Academy of Sciences, Beijing 100080, \\ People's Republic of China
}

(Received 19 February 2011; accepted 29 June 2011; published online 18 August 2011)

\begin{abstract}
Nonlinear optical properties of luminescent multinuclear platinum(II) complex of branched alkynyls in benzene solution are investigated at room temperature by using two-photon fluorescence (TPF) technique. It is found that the material shows unusual nonlinear optical characteristics under the excitation of near infrared femtosecond laser pulses. The self-focusing of laser beam energy during propagation of the laser pulses in the sample with large nonlinear coefficient for the refractive index is observed. Based on this phenomenon, a new method for measuring the nonlinear coefficient and two-photon absorption cross section of materials is proposed. (C) 2011 American Institute of Physics. [doi:10.1063/1.3622587]
\end{abstract}

\section{INTRODUCTION}

As one typical kind of nonlinear optical effect, two-photon absorption induced fluorescence (TPF) have many applications including two-photon microscopy, ${ }^{1,2}$ two-photon absorption spectroscopy, ${ }^{3,4}$ fluorescence correlation spectroscopy in living cells ${ }^{5,6}$ ultra-short laser pulse measurement, ${ }^{7,8}$ and optical power limiting, ${ }^{9,10}$ and three-dimensional optical data storage. ${ }^{11,12}$ Recently, we have investigated TPF processes in wide gap semiconductors ${ }^{13,14}$ and their quantum well structures ${ }^{15}$ using intense femtosecond near-infrared laser. For these solid state materials, their TPF exhibits a typical quadratic dependence on the excitation intensity. On the other hand, organic luminescent materials chemically synthesized have been extensively investigated in the past decades, and many new organometallic luminescent materials have been designed and synthesized. ${ }^{16,17}$ Nonlinear optical properties, such as, twophoton absorption, TPF etc., of these novel optoelectronic materials are emerging as a new frontier of multidisciplinary research.

Usually, two-photon fluorescence excited at low laser intensity obeys the quadratic intensity law. However, several groups have found deviations from this law. Using nanosecond laser pulses as excitation source, Hermann and Ducuing found deviations from the $I_{0}^{2}$ dependence in rhodamine $\mathrm{B} .{ }^{18}$ Here $I_{0}$ is average excitation laser intensity. Using picosecond excitation pulses, Bradley et al. reported deviations from the quadratic-intensity law in rhodamine B and rhodamine 6 G dye solutions. ${ }^{19}$ They suggested stimulated absorption and excited-state absorption as the cause for the deviation in rhodamine $6 \mathrm{G}$ and excited-state absorption in rhodamine B. Fischer, Cremer, and Stelzer also reported a significant

\footnotetext{
a)Present address: Bureau of Basic Research, Chinese Academy of Sciences, Beijing 100864, People's Republic of China.

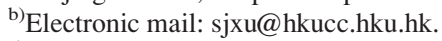

${ }^{\mathrm{c})}$ Electronic mail: wwyam@hku.hk.
}

departure from the quadratic-intensity dependence in rhodamine B and fluorescein using 80-130 fs laser pulses at a repetition rate of $82 \mathrm{MHz}$ as excitation source. ${ }^{20}$ Wang et al. also found the deviation of $I_{0}^{2}$ dependence at high laser intensity (about $10^{11} \mathrm{~W} / \mathrm{cm}^{2}$ ), and they introduced into a two-photon saturation intensity $I_{S}$ to explain this deviation. ${ }^{21}$ These works and different explanations indicate that the present understanding of departure, in particular, large departure of excitation-intensity dependence of TPF from the quadratic law is far from complete. In this paper, we present a study on the excitation-intensity dependence of the TPF signals from novel organometallic luminescent materials and suggest the self-focusing of laser pulses during the propagation in the solution samples as the physical origin causing the anomalous excitation-intensity dependence of the TPF signal.

\section{SAMPLES AND EXPERIMENTAL DETAILS}

In our experiment, the two-photon fluorescence spectra were measured using an experimental geometric configuration in which the fluorescence collecting direction is normal to the excitation beam. The samples were prepared from a branched platinum(II) alkynyl complex $\left[1,3,5-\left\{\mathrm{H}_{3} \mathrm{CC}_{6} \mathrm{H}_{4} \mathrm{C}\right.\right.$ $\left.\equiv \mathrm{C}\left(\mathrm{PEt}_{3}\right)_{2} \mathrm{PtC} \equiv \mathrm{CC}_{6} \mathrm{H}_{4} \mathrm{C} \equiv \mathrm{C}_{3} \mathrm{C}_{6} \mathrm{H}_{3}\right]$ in benzene solution. The molecular structure of the platinum complex was schematically shown in Fig. 1. The chemical synthesis procedures and detailed structures of the complexes have been previously reported elsewhere. ${ }^{16}$ The excitation laser was a tunable mode-locked Ti:Sapphire femtosecond laser with pulse width of $\sim 100$ fs (Spectra-Physics Tsunami system). The laser wavelength can be tuned from 700 to $930 \mathrm{~nm}$. The laser pulse was focused onto the sample solution in a quartz cell using a lens with $f=10 \mathrm{~cm}$. A circular variable metallic neutral density filter was used to change the intensity of excitation laser. In the direction perpendicular to the incident excitation laser, the fluorescence light emitted from sample was collected into an Acton SP350 monochromator by a 


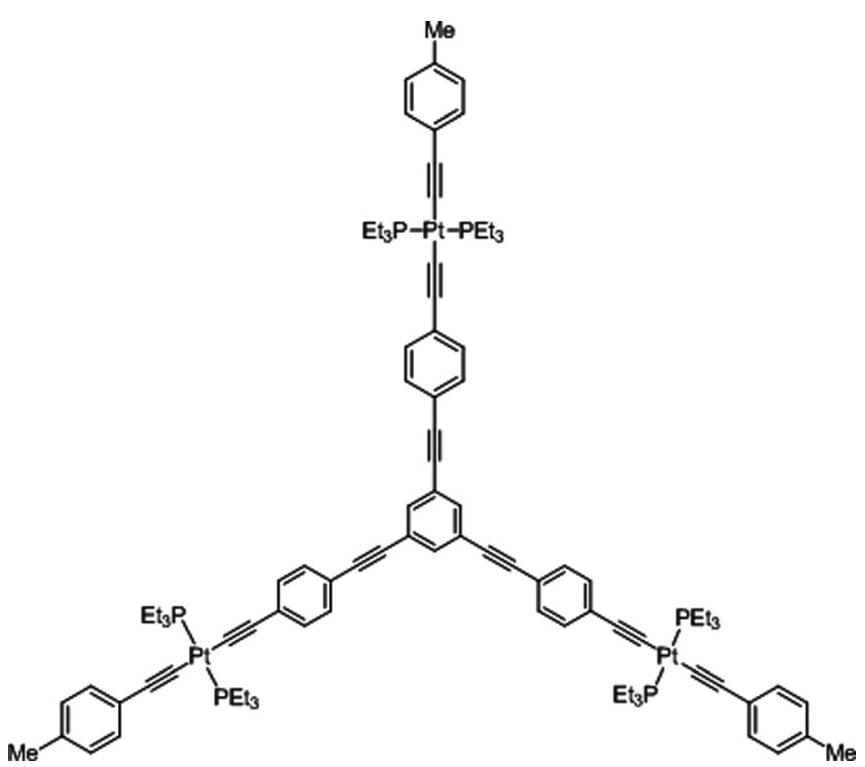

FIG. 1. Molecular structure of the platinum(II) complex.

telescope system and then detected by a Hamamatsu R928 photomultiplier. Standard lock-in amplification technique was employed in the TPF measurements.

\section{RESULTS AND DISCUSSION}

Because one-photon absorption peak of this sample is about $370 \mathrm{~nm}$, so we tuned the laser wavelength to $740 \mathrm{~nm}$ and measured the TPF spectra of the solution sample. Fig. 2 shows a typical TPF spectrum (solid line) from a solution sample with the concentration of branched platinum(II) alkynyl complex, $1.686 \times 10^{-4}$ M. One-photon emission spectrum (dashed line) of the sample was also given for comparison. It can be seen that the lineshapes of both spectra are almost identical, which means that the TPF stems from the same electronic transition as the one-photon fluorescence. However, the overall full width at half maximum height of both the spectra shows some difference. It is most likely due to the difference in coupling strength of electron-molecular vibration of the sample under the conditions of one- and

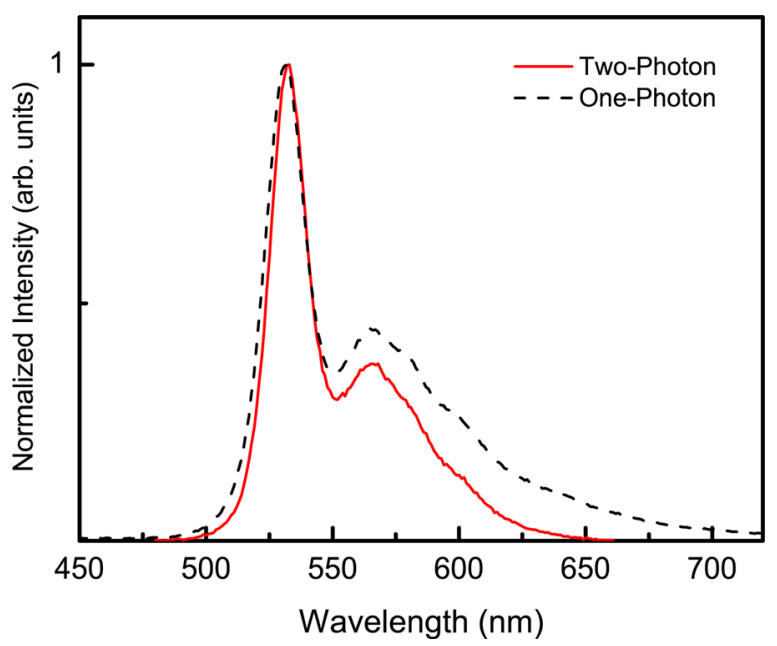

FIG. 2. (Color online) Two-photon and one-photon fluorescence spectra of the sample at room temperature. two-photon excitations. In addition, efficient self-absorption (reabsorption) of the fluorescence, especially the dominant narrow emission peak inside the sample under the excitation of two photons could be another physical mechanism causing the observed difference in the two excitation situations. Further experimental and theoretical studies need to be done for elucidating this phenomenon. It is more interesting that a green TPF luminescence channel with the cell length of about $10 \mathrm{~mm}$ rather than an emitting spotty was observed in the sample cell, as shown in Fig. 3(a). Three samples with different concentrations of luminescent materials, say $1.686 \times 10^{-4} \mathrm{M}, 6.744 \times 10^{-5} \mathrm{M}$, and $3.372 \times 10^{-5} \mathrm{M}$, were prepared to measure the power-dependence of sample TPF signals, respectively. At the same excitation power, as expected, the luminescent channel was observed to become brighter in intensity and wider in width for the samples with higher concentrations. On the other hand, for the individual samples, the luminescent channel tends to become thinner with decreasing power, as shown in Fig. 3(b). The concentration of branched platinum(II) alkynyl complex in the solution sample whose fluorescence images were shown in Fig. 3 was $6.744 \times 10^{-5} \mathrm{M}$. When the excitation power is becoming further weaker, the TPF emission can occur only around the focus point of lens. The bright streak bands in Fig. 3 are the images of the transmitted parts of the primary excitation beams. In Fig. 4, the power dependence of TPF of three samples was shown. From the figure, it is found that the dependence has large deviation from the theoretical quadratic relationship. In particular, for the sample with higher concentrations of luminescent molecules under high excitation, the deviation is very large. For example, as seen in Fig. 4(a), for the sample with concentration of $1.686 \times 10^{-4} \mathrm{M}$, the excitation-power dependence of its TPF is nearly linear (i.e., the slope is about 1.0.) at high excitation. As the excitation power is decreased, the slope slowly increases. From Figs. 4(b) and 4(c), we can clearly see that the power dependence changes from the almost linear relation to the quadratic relation with decreasing the excitation power.

Observation of the linear dependence of the TPF signal on the excitation power under the condition of high excitation is interesting. Here we attempt to attribute it to the selffocusing and self-trapping effects of intense laser pulses propagating in nonlinear media. ${ }^{22-24}$ As pointed out by Chiao, Garmire, and Townes in their classic paper, the selffocusing and trapping effects mean that an intense coherent

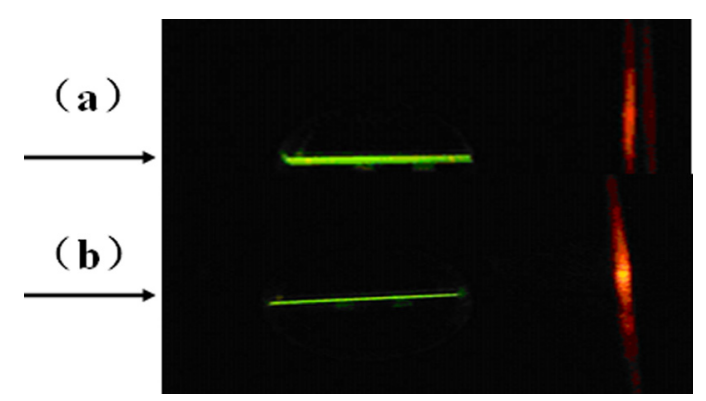

FIG. 3. (Color online) Photographs of the green luminescent channels in the solution sample cell with the concentration of $6.744 \times 10^{-5} \mathrm{M}$, (a) at the excitation power of $650 \mathrm{~mW}$, (b) $395 \mathrm{~mW}$. 

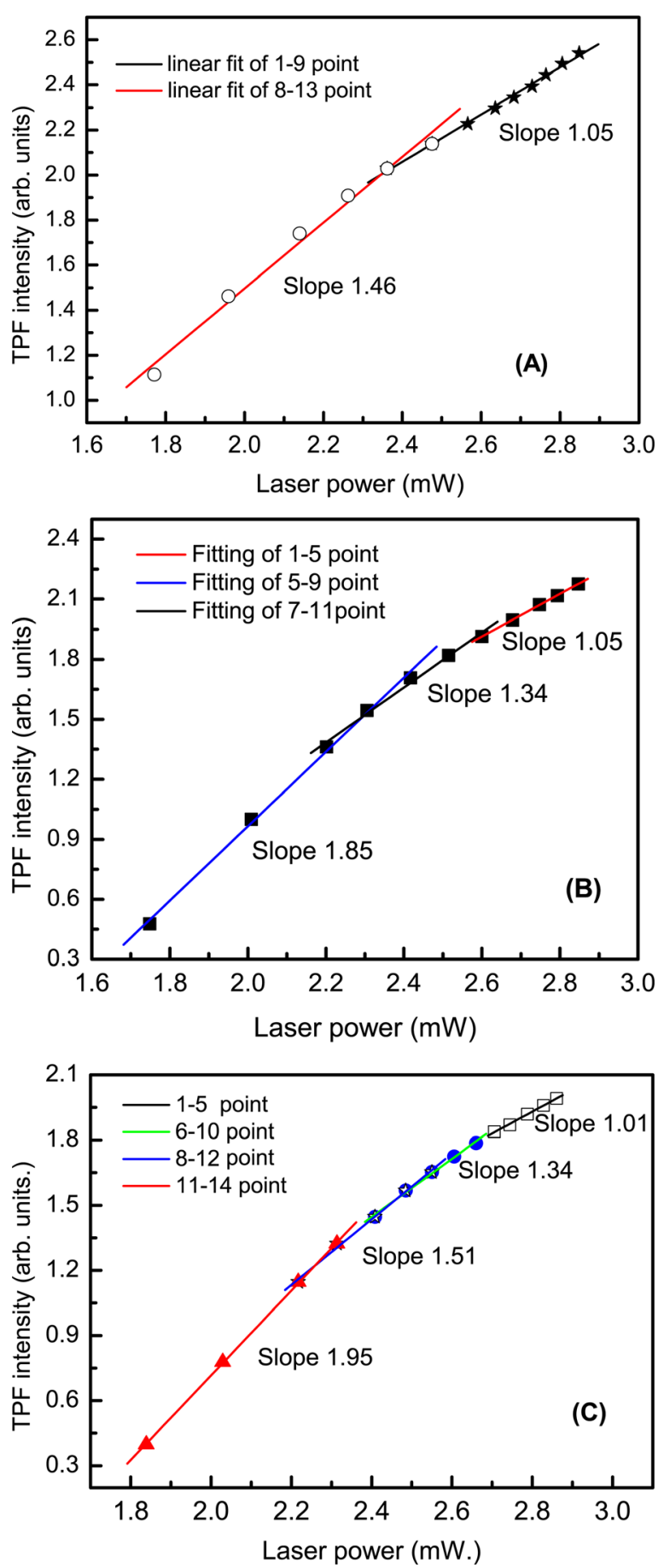

FIG. 4. (Color online) The excitation-intensity dependence of the TPF of the samples with three different concentrations (A) $1.686 \times 10^{-4} \mathrm{M}$; (B) $6.744 \times 10^{-5} \mathrm{M}$; and (C) $3.372 \times 10^{-5} \mathrm{M}$.

optical beam can produce its own dielectric waveguide and propagate without diffraction in a nonlinear medium. ${ }^{23}$ Formation of such self-focusing waveguide requires a certain critical beam intensity $I_{c}=(1.22 \lambda)^{2} c / 64 n_{2}$, where $\lambda$ is the wavelength of light, $c$ the light speed in vacuum, and $n_{2}$ the coefficient of the refractive index nonlinearity of material. The nonlinear coefficient $n_{2}$ may be associated with Kerr effects involving molecular orientation and with electrostrictive effects associated with the electrically induced mechanical deformation. Both the effects are of comparable size for liquid media whereas the latter dominates for solids. ${ }^{23}$ When laser pulses pass through a medium, the strong electric field of the laser pulses will lead to an intensity-dependent nonlinear refractive index $n=n_{0}+n_{2} I+\ldots$, where $n_{0}$ is a constant and $I$ is the laser intensity. The higher intensities the laser pulses the larger nonlinear refractive index they experience. The larger index in turn leads to further concentration of the laser intensity along the beam central line. ${ }^{25}$ Correspondingly, the effective cross area of the laser beam tends to become smaller with increasing the laser intensity. A crude estimation for the effective cross area of the laser beam with intensity above the critical value can be undertaken by $\pi R^{2} \approx(1.22 \lambda)^{2} \pi / 8 n_{0} n_{2} I .^{23}$ This means that the effective cross area of the laser beam is roughly proportional to the inverse of the laser intensity. For the fluorescence induced by two-photon absorption, its intensity can be written as $I_{T P F}=C_{g} V A_{r} N$, where $C_{g}$ is the luminescence collection factor depending on the details of experimental arrangement, $V=\pi R^{2} L$ ( $L$ is the laser beam channel length) the effective volume in which light emission generates, $A_{r}$ the radiative recombination rate, and $N$ the density of excited carriers. Assuming that the laser pulses have Gaussian shape, ${ }^{26}$ the average two-photon fluorescence intensity $I_{T P F}$ can be calculated by $I_{T P F}=C_{g} V A_{r} \beta T I^{2} / 2 \sqrt{\pi} h v \tau$, where $\beta$ is the twophoton absorption coefficient, $T$ is the period of the laser pulse train, $h v$ the photon energy of the laser beam, and $\tau$ the pulse width. Obviously, TPF intensity shall exhibit a quadratic dependence on the excitation intensity when the luminescent volume keeps unchanged. According to the discussion given earlier, however, when the excitation intensity is strong enough the self-focusing and self-trapping effects occur. As a result, the luminescent volume will decrease in a rate of the inverse of the excitation intensity. Thus the two-photon fluorescence could be linearly dependent on the excitation intensity. Indeed, the almost linear dependence of the TPF signal is observed in the solution sample containing the new Pt-based luminescent molecules at high excitation, as shown in Fig. 4. In order to verify that the observed abnormal power dependence of the TPF in the solution samples is really due to the self-focusing rather than due to others, we prepared solid state samples of the same luminescent materials and then measured the TPF of the solid state samples. It is firmly found that the dependence of the TPF signals of the solid-state samples satisfies quadratic law as shown in Fig. 5. We also found that the sample concentration affects the self-focusing of laser pulses propagating inside the sample. When the concentrations of samples are decreased from $6.744 \times 10^{-5} \mathrm{M}$ to $3.372 \times 10^{-5} \mathrm{M}$, more laser energy is required to keep the self-focusing. It is because that the nonlinear refraction indexes of the samples depend on the concentration of luminescent molecules.

From the experimental results, the nonlinear refractive index can be extracted. There exist three metal-nuclear centers $(\mathrm{Pt})$ in one luminescent molecule $\mathrm{e}^{16,17}$ in the samples studied in the present work. According to the plasma's selffocusing theory, ${ }^{22,23}$ the refractive index can be written as

$$
n=n_{0}+n_{2} I-\frac{n_{e}}{2 n_{c}},
$$




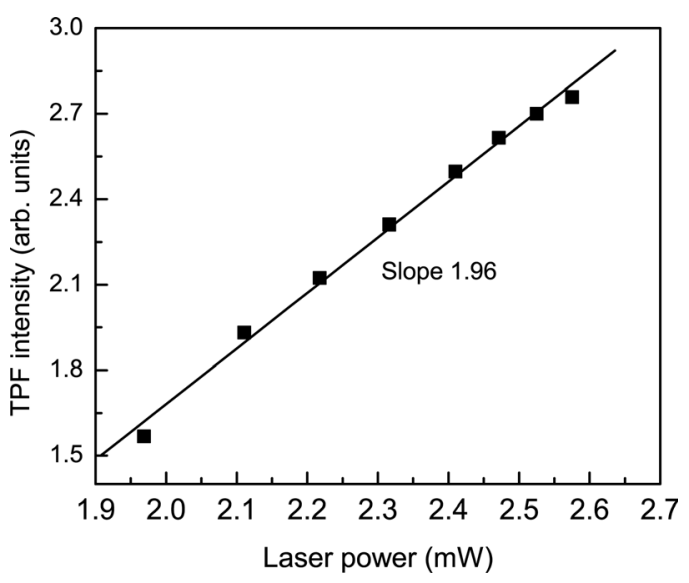

FIG. 5. The excitation-intensity dependence of the TPF in solid state sample.

where $n_{0}$ is the constant refractive index, $n_{2}$ is the nonlinear refractive index, $I$ is the laser intensity, $n_{e}$ is the electron density, and $n_{c}=\varepsilon_{0} m_{e} \omega^{2} / e^{2}$ is the plasma critical density. In our case, $n_{c}=2.0 \times 10^{21} / \mathrm{cm}^{3}$. The second term on the right-hand side of Eq. (1) represents the self-focusing while the third term stands for the defocusing. For the stable selffocusing, it should satisfy $n_{2} I_{c}=\frac{n_{e}}{2 n_{c}}$. So, $n_{2}=\frac{n_{e}}{2 n_{c} I_{c}}$. Assuming that one molecule can provide three electrons as there are three metal-nuclear centers for each molecule, we can estimate that the electron densities in the solution samples are about $3 \times 10^{17} / \mathrm{cm}^{3}, 1.2 \times 10^{17} / \mathrm{cm}^{3}, 0.60 \times 10^{17} / \mathrm{cm}^{3}$ for the concentrations of $1.686 \times 10^{-4} \mathrm{M}, 6.744 \times 10^{-5} \mathrm{M}$, and $3.372 \times 10^{-5} \mathrm{M}$, respectively. If considering the channel radius is about $50 \mu \mathrm{m}$ and the excitation laser intensity with $10 \%$ deviation from the linear relation as the critical intensity $I_{c}$, from Fig. 4, we can get $I_{c} \approx 1.4 \mathrm{GW} / \mathrm{cm}^{2}, 2.17$ $G W / \mathrm{cm}^{2}$, and $3.22 \mathrm{GW} / \mathrm{cm}^{2}$ (corresponding laser powers are about $220 \mathrm{~mW}, 350 \mathrm{~mW}$, and $520 \mathrm{~mW}$, respectively.) for the samples with the concentrations of $1.686 \times 10^{-4} \mathrm{M}$, $6.744 \times 10^{-5} \mathrm{M}$, and $3.372 \times 10^{-5} \mathrm{M}$, respectively. The corresponding nonlinear refractive indexes $n_{2}$ are then determined to be $5.0 \times 10^{-14} \mathrm{~cm}^{2} / \mathrm{W}, 1.4 \times 10^{-14} \mathrm{~cm}^{2} / \mathrm{W}$, and $4.7 \times 10^{-15} \mathrm{~cm}^{2} / \mathrm{W}$. Furthermore, if the stable self-focusing forms, the two-photon absorption cross sections $\sigma_{2}$ can be calculated from $N_{0} \sigma_{2}=h v \beta$, where $N_{0}$ is the concentration of luminescent molecules. Due to $\frac{d I}{d z}=-\beta I^{2}$ and considering laser intensity in channel is constant, so $\beta=-\frac{\Delta \varepsilon}{I_{c} L \varepsilon}$ (considering that it is absorption, $\Delta \varepsilon$ is negative). Here $\Delta \varepsilon$ is the energy difference between the input and output laser beams, which is easily detected due to the long luminescent channel. $L$ is the luminescent channel length. Therefore, an alternative new method can be used to measure two-photon absorption cross section $\sigma_{2}$, which is simpler and easier than the Z-scan method. For example, when input laser energy is about $600 \mathrm{~mW}$, the absorption energy is about $4.5 \mathrm{~mW}$ for the samples with the concentrations of $1.686 \times 10^{-4} \mathrm{M}$, so $\beta=2.3 \times 10^{-12} \mathrm{~cm} / \mathrm{W}$, and $\sigma_{2}=6.1 \times 10^{-48} \mathrm{~cm}^{4} \mathrm{~s}$.

\section{CONCLUSION}

In conclusion, an experimental study on the excitationintensity dependence of the two-photon absorption induced fluorescence in the new class of luminescent multinuclear platinum(II) complex of branched alkynyls at room temperature was presented. The linear dependence of the TPF on the excitation intensity was observed when the excitation intensity is strong enough. Based on the self-focusing effect in the solution samples, we explain the observed abnormal dependence of the TPF on the excitation intensity. Furthermore, a new method is proposed to measure the nonlinear refraction index and two-photon absorption cross section $\sigma_{2}$ of materials.

\section{ACKNOWLEDGMENTS}

The work was supported by the Joint Research Fund for Overseas Chinese, Hong Kong, and Macau Scientists of NSFC (Grant No. 61028012) and the HK RGC-CERG Grant (Grant No. HKU 7056/06P), and partially supported by a grant from the University Grants Committee Areas of Excellence Scheme of the Hong Kong Special Administrative Region, China (Project No. [AoE/P-03/08]).

${ }^{1}$ P. R. Monson and W. W. McClain, J. Chem. Phys. 53, 29 (1970).

${ }^{2}$ S. P. Tai, M. C. Chan, T. H. Tsai, S. H. Guo, L. J. Chen, and C. K. Sun, Opt. Express 12, 6122 (2004).

${ }^{3}$ W. Denk, J. H. Strickler, and W. W. Webb, Science 248, 73 (1990).

${ }^{4}$ V. E. Centonze and J. G. White, Biophys. J. 75, 2015 (1998).

${ }^{5}$ K. M. Berland, P. T. So, and E. Gratton, Biophys. J. 68, 694 (1995).

${ }^{6}$ P. Schwille, U. Haupts, S. Maiti, and W. W. Webb, Biophys. J. 77, 2251 (1999).

${ }^{7}$ A. M. Streltsov, K. D. Moll, A. L. Gaeta, P. Kung, D. Walker, and M. Razeghi, Appl. Phys. Lett. 75, 3778 (1999).

${ }^{8}$ M. Muller, J. Squier, and G. J. Brakenhoff, Opt. Lett. 20, 1038 (1995).

${ }^{9}$ G. S. He, J. D. Bhawalkar, C. F. Zhao, and P. N. Prasad, Appl. Phys. Lett. 67, 2433 (1995)

${ }^{10}$ J. E. Ehrlich, X. L. Wu, I.-Y. S. Lee, Z.-Y. Hu, H. Rockelm, S. R. Marder, and J. W. Perry, Opt. Lett. 22, 1843 (1997).

${ }^{11}$ J. H. Strickler and W. W. Webb, Opt. Lett. 16, 1780 (1991).

${ }^{12}$ D. A. Parthenopoulos and P. M. Rentzepis, Science 245, 843 (1989).

${ }^{13}$ D. C. Dai, S. J. Xu, S. L. Shi, M. H. Xie, and C. M. Che, Opt. Lett. 30, 3377 (2005).

${ }^{14}$ H. Yang, S. J. Xu, Q. Li, and J. Zhang, Appl. Phys. Lett. 88, 161113 (2006).

${ }^{15}$ Q. Li, S. J. Xu, G. Q. Li, D. C. Dai, and C. M. Che, Appl. Phys. Lett. 89, 011104 (2006).

${ }^{16}$ C.-H. Tao, N. Zhu, and V. W.-W. Yam, Chem. Eur. J. 11, 1647 (2005).

${ }^{17}$ C.-H. Tao, H. Yang, N. Zhu, V. W.-W. Yam, and S. J. Xu, Organometallics 27, 5453 (2008).

${ }^{18}$ J. P. Hermann and J. Ducuing, Phys. Rev. A 5, 2557 (1972).

${ }^{19}$ D. J. Bradley, M. H. R. Hutchinson, H. T. Koetser, C. Morrow, H. C. New, and M. S. Petty, Proc. R. Soc. London, Ser. A 328, 97 (1972).

${ }^{20}$ A. Fischer, C. Cremer, and E. H. Stelzer, Appl. Opt. 34, 1989 (1995).

${ }^{21}$ C. H. Wang, O. Y.-H. Tai, Y. Wang, T.-H. Tsai, and N.-C. Chang, J. Chem. Phys. 122, 084509 (2005).

${ }^{22}$ Y. R. Shen, The Principles of Nonlinear Optics (Academic, New York, 1993).

${ }^{23}$ R. Y. Chiao, E. Garmire, and C. H. Townes, Phys. Rev. Lett. 13, 479 (1964).

${ }^{24}$ H. Yang, J. Zhang, W. Yu, Y. J. Li, and Z. Y. Wei, Phys. Rev. E 65, 016406 (2002).

${ }^{25}$ H. A. Haus, Appl. Phys. Lett. 8, 128 (1966).

${ }^{26}$ Z. Zheng, A. M. Weiner, J. H. Marsh, and M. M. Karkhanehchi, IEEE Photo. Tech. Lett. 9, 493 (1997). 